\title{
Judicious Use of Elective Surgical Lists for Emergency Cholecystectomies for Acute Cholecystitis can Improve Quality of Care in Systems with Limited Resources
}

\author{
Nitya Krishnamohan ${ }^{1}$, Claudia Koh ${ }^{1}$, Yen Yeen Teh ${ }^{1}$, Jeremy Bruce Ward ${ }^{1}$ and Ravindra Sudhachandra Date ${ }^{1,2^{\star}}$ \\ ${ }^{1}$ Department of Upper GI Surgery, Lancashire Teaching Hospitals NHS Foundation Trust, United Kingdom \\ ${ }^{2}$ The University of Manchester, Manchester Academic Health Science Centre, United Kingdom
}

"Corresponding author: Ravindra Sudhachandra Date, Department of Upper GI Surgery, Lancashire Teaching Hospitals NHS Foundation Trust, United Kingdom, Tel: +441257245267; E-mail: ravidate@hotmail.com

Received date: August 22, 2018; Accepted date: September 7, 2018; Published date: September 14, 2018

Copyright: (c) 2018 Krishnamohan N, et al. This is an open-access article distributed under the terms of the Creative Commons Attribution License, which permits unrestricted use, distribution and reproduction in any medium, provided the original author and source are credited.

\begin{abstract}
Background: NHS hospitals currently have limited capacity in emergency theatres for emergency Laparoscopic Cholecystectomy (LC). A pathway was introduced in this Trust allowing for emergency LC to be performed on an elective operating list. This study aims to assess its impact on patient care.

Methods: Acute admissions with biliary complaints from April to September 2014 were identified prospectively (Group 1). Mode of referral for patients undergoing elective LC during the same period along with other data was collected retrospectively (Group 2). The two groups were compared for readmission rates, length of hospital stay (LOS) and conversions.

Results: Of the 207 acute admissions, 115 (56\%) were eligible for emergency surgery. Thirty-three patients $(28.7 \%)$ had emergency surgery; 20 in emergency theatre and 13 on the pathway. One of 13 was converted to an open procedure. Average LOS for these 13 patients was 8 days. $11(13.4 \%)$ of the remaining 82 patients were readmitted whilst awaiting surgery.

Of the 131 patients undergoing elective LC, $38(29 \%)$ were listed for surgery following acute admission prior to introduction of the pathway. Five of the $38(13.1 \%)$ had readmissions whilst awaiting surgery, but none required conversion to open surgery. Average LOS for these 5 patients (including previous readmissions) was 6 days and that for 38 patients was 8 days.

Conclusions: The use of elective lists to perform emergency LC is a feasible option. This model of care has facilitated participation in the Chole-QulC initiative. Expansion and sustained use of this model has enabled more patients to undergo emergency LC. It may prevent readmission in those undergoing delayed LC, although its impact on total LOS, other elective surgeries and conversions remains to be assessed.
\end{abstract}

Keywords: Acute cholecystitis; Emergency cholecystectomy; Elective

Abbreviations: AC: Acute Cholecystitis; AGP: Acute Gallstone Pancreatitis; Chole-QuIC: Cholecystectomy Quality Improvement Collaborative; IAP: International Association of Pancreatology; IHPBA: International Hepato-Pancreato-Biliary Association; LC: Laparoscopic Cholecystectomy; LOS: Length of hospital Stay; NCEPOD: National Confidential Enquiry into Patient Outcome and Death; NICE: National Institute for Health and Care Excellence

\section{Introduction}

Tokyo Guidelines from the International Hepato-Pancreato-Biliary Association (IHPBA) advocates Laparoscopic Cholecystectomy (LC) during index admission, especially within 72 hours of symptom onset [1] and National Institute for Health and Care Excellence (NICE) guidance recommends LC within 7 days for acute cholecystitis (AC) [2]. The International Association of Pancreatology (IAP) and National Confidential Enquiry into Patient Outcome and Death (NCEPOD) advise LC for Acute Gallstone Pancreatitis (AGP) during the same admission $[3,4]$. The British Society of Gastroenterology suggests LC at least within 14 days of diagnosis for AGP [5].

In many NHS hospitals currently, emergency cholecystectomy for AC or AGP is not performed within this time frame [6]. Implementation of these guidelines is difficult in many hospitals due to limited capacity in emergency theatres and non-availability of experienced surgeons to perform emergency LC. This is in contrast to practice in other developed countries where LC is offered as soon as possible to the patients with AC [7].

In this Trust, majority of patients presenting with $\mathrm{AC}$ were treated conservatively and then offered a delayed LC on an elective list. This was not complying with current recommendations and hence to improve the quality of care provided to these patients within given resources following approach was considered on ad hoc basis in 2011. Rather than using the time slot on an elective list for a delayed LC, it was decided to offer this slot to the patients with AC on one elective list every week. To formalise this concept, a pathway was introduced in the 
Citation: Krishnamohan N, Koh C, Teh YY, Ward JB, Date RS (2018) Judicious Use of Elective Surgical Lists for Emergency Cholecystectomies for Acute Cholecystitis can Improve Quality of Care in Systems with Limited Resources. Surgery Curr Res 8: 310. doi: $10.4172 / 2161-1076.1000310$

Page 2 of 4

Trust in April 2014, allowing for emergency LC to be performed on an elective operating list (Figure 1).

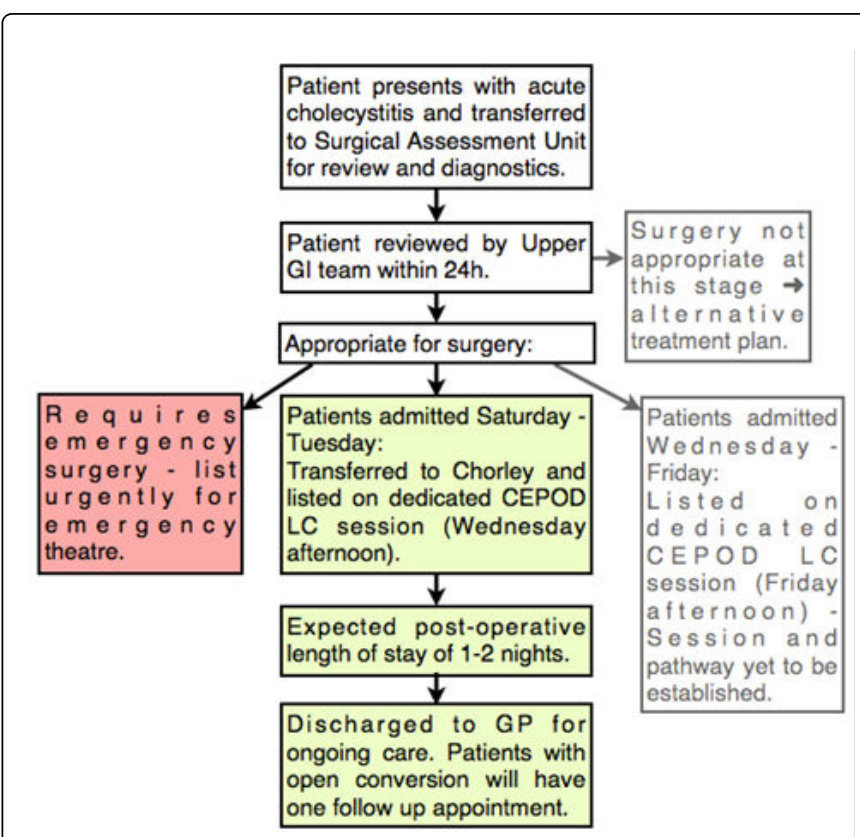

Figure 1: Pathway for patients presenting acutely with biliary complaints in the trust.

This study aims to assess the impact of the pathway on patient care by comparing different outcome measures for two groups of patients, namely those undergoing emergency LC on the new pathway (either at the acute site or the elective one; Group 1) and those referred for delayed LC from admissions prior to introduction of the pathway (Group 2).

\section{Methods}

We identified patients prospectively presenting with acute biliary symptoms, from April to September 2014. These patients were put on a pathway as illustrated in Figure 1. This Trust has two hospitals on different sites located 14 miles apart. The bigger hospital works as an acute site where all acute surgical patients are admitted. The other site works as an elective surgical centre where Upper GI and other specialties perform elective inpatient and day case operations. Within our Upper GI service, elective cases from day case LC to complex benign Upper GI operations are performed at this elective site. This site has an intensive care unit for post-operative care should the unscheduled need arise. Post-operative care during daytime working hours on weekdays is provided by the surgeon. A resident medical officer is available overnight and on weekends to provide patient care. The surgical registrar on-call at the acute site is available for contact out-of-hours for advice and decisions regarding transfer back to the acute site post-operatively should this become necessary. On the pathway, patients are categorised into either needing emergency surgery at the acute site, or if they are well enough, are transferred to the elective hospital site for an operation on a dedicated elective operating list provided by one surgeon. Only the patients admitted from Saturday onwards were accepted for an operation on Wednesday's elective list. If a suitable patient was not identified, then a "standby" patient was given the operation slot on the day before. The data were also collected retrospectively on patients undergoing delayed LC during the same period.

In Group 1, data were collected on diagnosis and outcome from the index admission including Pre- and post-operative Length of Stay (LOS) and conversion rates. This group was subdivided into those undergoing surgery on the pathway (Group 1a) and those having delayed surgery (Group 1b).

The same data were collected for patients in Group 2. Readmission rates whilst awaiting surgery, total LOS (including both pre- and postoperative) and conversions were compared for all the groups.

\section{Results \\ Group 1}

Of the 207 acute biliary admissions including biliary colic, AC, obstructive jaundice and AGP, 115 (56\%) were eligible for emergency surgery (Group 1). Patients were deemed unsuitable for emergency LC for various reasons (both medical and non-medical), such as:

- Unfit for surgery

- The slot was available more than 72 hours after admission

- Further investigations required (for example common bile duct stones)

- Gallstone pancreatitis, (these patients were excluded as they were given priority slots on all the lists even before the introduction of pathway)

- Patient refusal or self-discharge

Thirty-three patients (28.7\%) had emergency surgery; 20 in emergency theatre and 13 on the pathway. One of the 13 patients on the pathway was converted to an open procedure, and the average LOS for these 13 patients was 8 days. Of the remaining 82 patients, 11 (13.4\%) were readmitted whilst awaiting delayed surgery.

\section{Group 2}

Of the 131 patients undergoing an elective LC, 38 (29\%) were listed for surgery following acute admission prior to introduction of the pathway (delayed LC, Group 2). Five of the 38 (13.1\%) had readmissions whilst awaiting surgery, making their average LOS 6 days (including previous readmissions). None of the 38 patients required conversion to open surgery, and the average LOS was 8 days. These results are summarised in Figures 2 and 3.

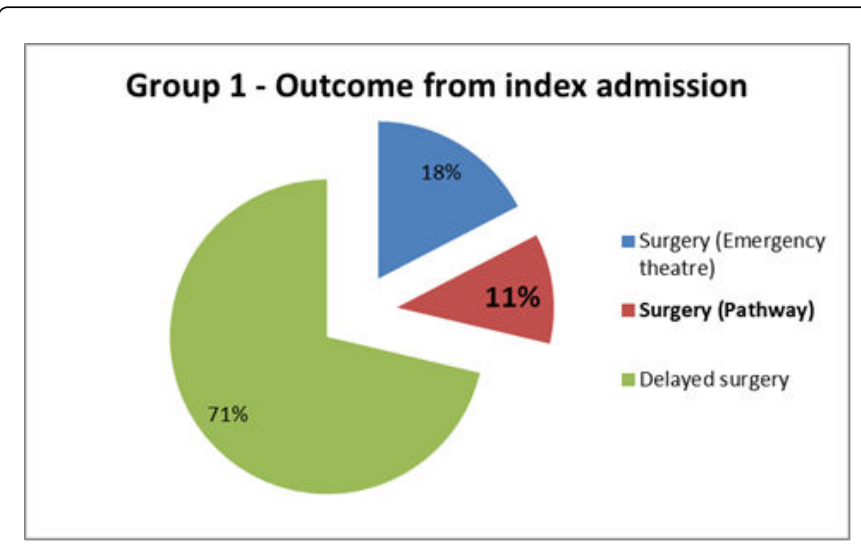

Figure 2: Group 1: Outcome from index admission. 
Citation: Krishnamohan N, Koh C, Teh YY, Ward JB, Date RS (2018) Judicious Use of Elective Surgical Lists for Emergency Cholecystectomies for Acute Cholecystitis can Improve Quality of Care in Systems with Limited Resources. Surgery Curr Res 8: 310. doi: $10.4172 / 2161-1076.1000310$

Page 3 of 4

\section{Group 2 - Elective Surgery}

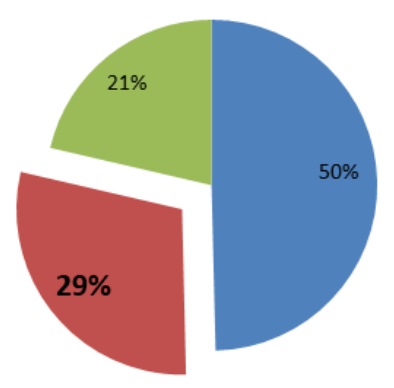

- GP Referral

nelayed surgery

non-GP referral

Figure 3: Group 2: Referral pattern for elective laparoscopic cholecystectomy.

\section{Discussion}

Delayed LC forms a significant proportion of elective theatre lists (29\% of all the elective LC in this study: Group 2). In this study, instead of a delayed LC, the patients are offered an emergency LC on the elective lists. The study confirms feasibility of using the elective lists for emergency LC.

Among various NHS hospitals, there remains the challenge of meeting the demand of rising number of emergency LC and meeting guidelines due to various factors such as theatre and bed capacity. Some hospitals have created a dedicated CEPOD list to deal with this. Other hospitals perform emergency LC on the daily CEPOD list. Some have a dedicated all day list for emergency LC. Each of these models has its pros and cons. Our study proposes another model of care that can be adopted for performing emergency LC. Our model works within existing resources and simply utilises the slot for delayed LC to do emergency LC. It also eliminates the aspect of 'unpredictability' that can occur on the CEPOD list where it is not uncommon that an emergency laparotomy, trauma or an emergency from another specialty takes priority. Our model provides a dedicated slot to the patient. This provides advantages to the patient as it deals with their acute biliary problem and secondly optimises the surgical service delivered.

A recent randomised trial comparing early versus delayed cholecystectomy showed an early cholecystectomy is still better than a delayed one, even for AC with more than 72 hours of symptoms [8]. We still believe emergency LC should be performed within 72 hours of acute presentation of AC. This view is supported by large retrospective population based studies showing higher morbidity, mortality, length of stay, and higher hospital costs in patients operated after 72 hours $[9,10]$.

The initiative to perform emergency LC on elective lists was initially started in the Trust on an ad hoc basis in 2011 and subsequently formalised as a pathway in 2014; years prior to the introduction of the Royal College of Surgeons of England's Cholecystectomy Quality Improvement Collaborative (Chole-QuIC) initiative [11]. The pathway was used only on a one elective list. The patients admitted within previous 3 days were operated on this list thus leaving patients admitted on other days disadvantaged. The limited capacity of one slot per session on the designated list has only allowed a small number of patients to have surgery on the pathway. Extension of the pathway to another list was considered so as to provide emergency LC to all AC patients.

A recent boost to the pathway came from the Chole-QuIC initiative that aims to achieve emergency cholecystectomy in $80 \%$ of eligible patients within 8 days (not 72 hours as previously used in the pathway) of presentation [11]. The model used in this study was implemented by the Trust to participate in the study. For this study, all the operating lists in the elective hospital were used for emergency LC with 2 dedicated slots on each list. The indications for eligibility on the pathway were also extended to the AGP patients. Our raw data from April 2016 to July 2017 (including first 6 months of the Chole-QuIC study) shows 304 acute hospital admissions with gallstone related diseases. Of these, 106 patients had AC and were eligible for emergency LC. 86 patients underwent an emergency LC within 8 days of admission-34 on the pathway at the elective site, the remaining 52 at the acute site and 20 had to have a delayed LC for various reasons. If we were working on single site then an additional 52 emergency LC could have been accommodated on the pathway. The impact of the pathway on elective surgical lists, of LC as well as other benign surgeries remains to be seen.

The numbers in this study are small to derive any meaningful outcome in terms of LOS or conversion rates and readmission rates, but these outcomes are unlikely to be different from previously published larger studies comparing early against delayed surgery for LC.

\section{Conclusion}

Our study proposes another model of care of performing emergency LC. Implementation of this model on two sites needs a dedicated "waiting list team" to coordinate between two sites and also good understanding between other teams involved including surgeons, anesthetists and preoperative assessment team. Our study shows that this is a feasible option. This model can be adapted for use in hospital trusts working on a single site where it is likely to work better.

\section{Declarations}

Ethics approval and consent to participate: Discussed with the local Research and Development department at Lancashire Teaching Hospitals NHS Foundation Trust. The study was classified as service evaluation. Hence, ethics approval and consent were waived.

\section{Conflict of Interest}

The authors disclosed no potential conflicts of interest.

\section{Authors' Contributions}

Nitya Krishnamohan was involved in revision drafting of the manuscript. Claudia Koh and Yen Yeen Teh were involved in data collection and analysis. Jeremy Bruce Ward was involved in concept and final approval of manuscript. Ravindra Sudhachandra Date was involved in design, concept, data analysis and final approval of manuscript. All authors read and approved the final manuscript. 
Citation: Krishnamohan N, Koh C, Teh YY, Ward JB, Date RS (2018) Judicious Use of Elective Surgical Lists for Emergency Cholecystectomies for Acute Cholecystitis can Improve Quality of Care in Systems with Limited Resources. Surgery Curr Res 8: 310. doi: $10.4172 / 2161-1076.1000310$

Page 4 of 4

\section{References}

1. Yamashita Y, Takada T, Strasberg SM, Pitt HA, Gouma DJ, et al. (2013) TG13 surgical management of acute cholecystitis. J Hepatobiliary Pancreat Sci 20: 89-96.

2. NICE guideline (CG188) (2014). Gallstone disease: Diagnosis and management.

3. Working Group IAP/APA Acute pancreatitis guidelines (2013). IAP/APA evidence:based guidelines for the management of acute pancreatitis. Pancreatology 13: e1-15.

4. NCEPOD (2016) Treat the cause: A review of the quality of care provided to patients treated for acute pancreatitis.

5. Working party of the British Society of Gastroenterology (2005). Association of surgeons of Great Britain and Ireland, Pancreatic Society of Great Britain and Ireland, and Association of Upper GI Surgeons of Great Britain and Ireland. UK guidelines for the management of acute pancreatitis. Gut 54 (S3): iiil-9.
6. CholeS Study Group, West Midlands Research Collaborative (2016) Population: based cohort study of outcomes following cholecystectomy for benign gallbladder diseases. Br J Surg 103: 1704-1715.

7. Lee SW, Yang SS, Chang CS, Yeh HJ (2009) Impact of the Tokyo guidelines on the management of patients with acute calculous cholecystitis. J Gastroenterol Hepatol 24: 1857-1861.

8. Roulin D, Saadi A, Di Mare L, Demartines N, Halkic N (2016) Early versus delayed cholecystectomy for acute cholecystitis, are the 72 hours still the rule?: A randomized trial. Ann Surg 264: 717-722.

9. Zafar SN, Obirieze A, Adesibikan B, Cornwell EE, Fullum TM, et al. (2015) Optimal time for early laparoscopic cholecystectomy for acute cholecystitis. JAMA Surg 150: 129-136.

10. Polo M, Duclos A, Polazzi S, Payet C, Lifante JC, et al. (2015) Acute cholecystitis:optimal timing for early cholecystectomy: A French nationwide study. J Gastrointest Surg 19: 2003-2010.

11. Abercrombie J (2017) Cholecystectomy quality improvement collaborative (Chole-QuIC). Royal College of Surgeons of England. 